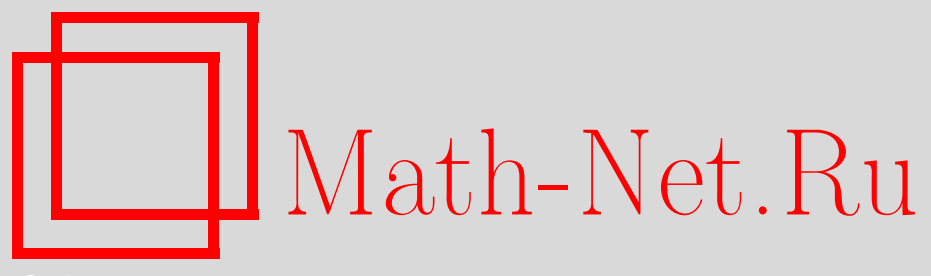

А. А. Пекарский, Об устранении особенностей мероморфных функций с конечным числом полюсов $p^{r}, M a$ тем. заметки, 2006, том 80, выпуск 2, 317-319

DOI: https://doi.org/10.4213/mzm2813

Использование Общероссийского математического портала Math-Net.Ru подразумевает, что вы прочитали и согласны с пользовательским соглашением http://www . mathnet.ru/rus/agreement

Параметры загрузки:

IP : 44.207 .124 .84

26 апреля 2023 г., 02:18:25

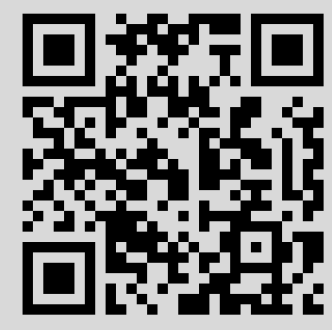




\section{ОБ УСТРАНЕНИИ ОСОБЕННОСТЕЙ МЕРОМОРФНЫХ ФУНКЦИЙ С КОНЕЧНЫМ ЧИСЛОМ ПОЛЮСОВ $p^{r}$}

\section{А. А. Пекарский}

Пусть $G$ - односвязная ограниченная область Жордана в комплексной плоскости; $\partial G$ граница $G$ и $\bar{G}=G \cup \partial G$. Через $E_{\infty}=E_{\infty}(G)$ обозначим пространство ограниченных аналитических функций $f$ в $G$, наделенных стандартной sup-нормой: $\|f\|_{E_{\infty}}=\sup _{z \in G}|f(z)|$. Далее $C_{A}=C_{A}(\bar{G})$ - подпространство $E_{\infty}$, состоящее из функций $f$, допускающих непрерывное продолжение в $\bar{G}$. В дальнейшем под $f(\xi)$ для $f \in C_{A}$ и $\xi \in \partial G$ подразумеваем $\lim _{G \ni z \rightarrow \xi} f(z)$. Полагаем $\|f\|_{C_{A}}=\|f\|_{E_{\infty}}$. В силу принципа максимума модуля аналитической функции имеем $\|f\|_{C_{A}}=\max _{\xi \in \partial G}|f(\xi)|$. Через $M=M(G)$ обозначим множество функций $h$, определенных в $G$ и представимых в виде $h=f-g$, где $f \in E_{\infty}$, а $g-$ рациональная функция (р. ф.) с полюсами лишь в $G$. Ясно, что $M$ есть некоторое подмножество множества мероморфных в $G$ функций, имеющих конечное число полюсов. Для $h \in M$ положим

$$
\|h\|_{M}=\sup _{\xi \in \partial G} \varlimsup_{G \ni z \rightarrow \xi}|h(z)| .
$$

Согласно результату Данченко [1], если $f \in E_{\infty}$ и $g_{m}-$ р. ф. степени $m \geqslant 1$ с полюсами лишь в $G$, то

$$
\|f\|_{E_{\infty}} \leqslant 51 m\left\|f-g_{m}\right\|_{M} .
$$

Оценке (1) предшествовали результаты Гончара и Григоряна [2], [3] и некоторых других авторов. Из примеров, построенных в [3], следует, что неравенство (1) точное в смысле порядка. Отметим также, хотя это мы и не будем использовать, что в [1] неравенство (1) получено для любой области.

В настоящей заметке мы получим одно дополнение к оценке (1). Именно, мы выясним: как хорошо можно исправить функцию $f$ посредством рациональных функций степени не выше $k, 1 \leqslant k \leqslant m$ ? При этом мы вынуждены на область $G$ накладывать дополнительные ограничения: $G$ должна быть областью Альпера или Радона. Определения этих областей имеются, например, в [4], [5], где даны также ссылки на другие работы. Условимся в дальнейшем через $c, c_{1}, c_{2}, \ldots$ обозначать некоторые положительные величины, зависящие лишь от $G$.

Теорема 1. Пусть $G$ - область Альпера или Радона, $f \in E_{\infty}(G), g_{m}-p . \oint$. степени $m, m \geqslant 1$, все полюсы которой лежат в $G u 1 \leqslant k \leqslant m$. Тогда

(i) существует p. $\oint . g_{k}$ степени не выше $k$ с полюсами лишь в $G$ такал, что

$$
\left\|f-g_{k}\right\|_{M} \leqslant \frac{c_{1} m}{k}\left\|f-g_{m}\right\|_{M}
$$

(ii) существует $p . \phi . \omega_{k}$ степени не выше $k$ с полюсами лишь в области $\Omega=\overline{\mathbb{C}} \backslash \bar{G}$ такая, что

$$
\left\|f-\omega_{k}\right\|_{E_{\infty}} \leqslant \frac{c_{2} m}{k}\left\|f-g_{m}\right\|_{M} .
$$

Для $f \in C_{A}(\bar{G})$ введем наилучшие равномерные рациональные приближения:

$$
R_{n}(f, \bar{G})=\inf _{r_{n}} \max _{z \in \bar{G}}\left|f(z)-r_{n}(z)\right|, \quad R_{n}(f, \partial G)=\inf _{r_{n}} \max _{\xi \in \partial G}\left|f(\xi)-r_{n}(\xi)\right|,
$$

где инфимумы берутся по всем рациональным функциям $r_{n}$ степени не выше $n, n=$ $0,1,2, \ldots$. При вычислении $R_{n}(f, \bar{G})$ мы должны учитывать р. ф. с полюсами лишь в области $\Omega$, а при вычислении $R_{n}(f, \partial G)$ - в $G \cup \Omega$. Поэтому $R_{n}(f, \partial G) \leqslant R_{n}(f, \bar{G})$.

(C) А. А. ПЕКАРСКий, 2006 
Поскольку полюсы дроби наилучшего приближения для $R_{n}(f, \partial G)$ могут оказаться в $G$, то, вообще говоря, последнее неравенство нельзя заменить равенством. Правда, это неравенство можно частично обратить. Именно, из (1) немедленно следует, что

$$
R_{n}(f, \bar{G}) \leqslant 51 n R_{n}(f, \partial G), \quad n \geqslant 1 .
$$

Из теоремы 1 (ii) легко получить, что при дополнительных ограничениях на $G$ множитель $51 n$ в (2) можно заменить некоторой постоянной, зависящей от области $G$.

Теорема 2. Если $G$ - область Альпера или Радона $u f \in C_{A}(\bar{G})$, то для $n=1,2$, $3, \ldots$ выполняется неравенство

$$
R_{n}(f, \bar{G}) \leqslant c \cdot R_{n}(f, \partial G) .
$$

Отметим, что неравенство типа (2) было впервые найдено Гончаром и Григоряном [2], [3]. Теорема 2 для круга получена нами ранее (см. [4] или [6; с. 313]) с константой $c=2$.

При доказательстве теоремы 1 мы будем применять классические пространство Лебега $L_{p}$ на $\partial G$; пространство Харди $H_{p}$ в круге $D=\{w:|w|<1\}$; пространства В. И. Смирнова $E_{p}$ в областях $G$ и $\Omega$. Будем считать $1 \leqslant p \leqslant \infty$ и, что эти пространства наделены стандартными нормами. Необходимые сведения о пространствах Харди и Смирнова можно найти, например, в монографиях [6], [7], а также в работах [5], [8].

ДоКАЗАТЕЛЬСтво теоремы 1. Положим $a=\left\|f-g_{m}\right\|_{M}$. Согласно теореме 3.1 из [4], или теореме 3 из [1], или теореме 4.1 из [8] имеют место соотношения

$$
\left\|g_{m}^{\prime}\right\|_{E_{1}(\Omega)}=\left\|g_{m}^{\prime}\right\|_{L_{1}(\partial G)} \leqslant c_{1} m a .
$$

Поэтому ввиду теоремы 1.3 из [5] найдется р. ф. $g_{k}$ степени не выше $k, 1 \leqslant k \leqslant m$, с полюсами лишь в $G$ такая, что

$$
\left\|g_{m}-g_{k}\right\|_{M}=\left\|g_{m}-g_{k}\right\|_{C_{A}(\bar{\Omega})} \leqslant \frac{c_{2}}{k}\left\|g_{m}^{\prime}\right\|_{E_{1}(\Omega)} \leqslant \frac{c_{3} m a}{k} .
$$

Покажем, что функция $g_{k}$ удовлетворяет условию (i). Действительно,

$$
\left\|f-g_{k}\right\|_{M} \leqslant\left\|f-g_{m}\right\|_{M}+\left\|g_{m}-g_{k}\right\|_{M} \leqslant a+\frac{c_{3} m a}{k} \leqslant \frac{c_{4} m a}{k} .
$$

Для доказательства утверждения (ii) введем дополнительные обозначения. Через $z=\varphi(w)$ обозначим какую-либо функцию, осуществляющую конформное отображение круга $D$ на область $G ; w=\psi(z)$ - обратное отображение. Как известно [7], при условии спрямляемости $\partial G$ (в рассматриваемом случае $\partial G$ спрямляема), имеем $\varphi \in C_{A}(\bar{D})$, $\psi \in C_{A}(\bar{G}), \varphi^{\prime} \in H_{1}$ и $\psi^{\prime} \in E_{1}$.

Пусть $z_{1}, z_{2}, \ldots, z_{m}$ - полюсы $g_{m}$ (не ограничивая общности, будем считать их попарно различными). Положим $w_{k}=\psi\left(z_{k}\right)$ и введем произведение Бляшке порядка $m$ :

$$
B_{m}(w)=\prod_{k=1}^{m} \frac{w-w_{k}}{1-\bar{w}_{k} w} .
$$

Функция

$$
F(w):=\left\{f[\varphi(w)]-g_{m}[\varphi(w)]\right\} B_{m}(w) \in H_{\infty}
$$

и $\|F\|_{H_{\infty}}=\left\|f-g_{m}\right\|_{M}=a$. Из теоремы Пика-Неванлинны (см., например, [6; с. 592]) следует существование произведения Бляшке $B_{m-1}(w)$ порядка не выше $m-1$ и числа $\alpha$, $|\alpha| \leqslant a$, таких, что

$$
F\left(w_{k}\right)-\alpha B_{m-1}\left(w_{k}\right)=0 \quad \text { при } \quad k=1,2, \ldots, m .
$$


Значит, функция

$$
\lambda(z)=\frac{F[\psi(z)]-\alpha B_{m-1}[\psi(z)]}{B_{m}[\psi(z)]}=f(z)-g_{m}(z)-\alpha \frac{B_{m-1}[\psi(z)]}{B_{m}[\psi(z)]}
$$

принадлежит $E_{\infty}(G)$ и $\|\lambda\|_{E_{\infty}} \leqslant 2 a$. Введем в рассмотрение функцию

$$
t(z)=\alpha \frac{B_{m-1}[\psi(z)]}{B_{m}[\psi(z)]} \in M(G) .
$$

Поскольку $f(z)$ и $\lambda(z)$ аналитичны в $G$, то функция $u(z)=t(z)+g_{m}(z)$ также аналитична в $G$ и, более того, принадлежит $C_{A}(\bar{G})$. Легко показать, что

$$
\left\|t^{\prime}\right\|_{L_{1}(\partial G)} \leqslant 2 \pi|\alpha|(2 m-1) \leqslant 4 \pi m a .
$$

С учетом неравенства (3), получаем

$$
\left\|u^{\prime}\right\|_{E_{1}(G)}=\left\|u^{\prime}\right\|_{L_{1}(\partial G)} \leqslant\left\|t^{\prime}\right\|_{L_{1}(\partial G)}+\left\|g_{m}^{\prime}\right\|_{L_{1}(\partial G)} \leqslant c_{5} m a .
$$

Таким образом, $u^{\prime} \in E_{1}(G)$ и $\left\|u^{\prime}\right\|_{E_{1}(G)} \leqslant c_{5} m a$. Как и при доказательстве утверждения (i), снова применим теорему 1.3 из [5], однако, в данном случае для области $\Omega$. Согласно этой теореме существует р. ф. $\omega_{k}$ степени не выше $k$ с полюсами лишь в $\Omega$ и такая, что

$$
\left\|u-\omega_{k}\right\|_{C_{A}(\bar{G})} \leqslant \frac{c_{6} a m}{k} .
$$

Покажем, что $\omega_{k}$ удовлетворяет условию (ii). Действительно,

$$
\left\|f-\omega_{k}\right\|_{E_{\infty}(G)} \leqslant\|f-u\|_{E_{\infty}(G)}+\left\|u-\omega_{k}\right\|_{E_{\infty}(G)} \leqslant 2 a+\frac{c_{6} a m}{k} \leqslant \frac{c_{7} a m}{k} .
$$

Здесь мы применили неравенство (4), а также соотношения $f(z)-u(z)=\lambda(z)$ и $\|\lambda\|_{E_{\infty}} \leqslant$ 2a. Теорема 1 доказана.

Можно показать, что множитель $m / k$ в теореме 1 (i), (ii) является точным в смысле порядка. Соответствующий пример строится по аналогии с примером из [3], подтверждающим точность оценки (1).

Как отмечено выше, неравенство (1) выполняется для любой области $G$. Напротив же, наше дополнение к этому неравенству - теорема 1 получено при весьма жестких ограничениях: $G$ должна быть областью Альпера или Радона. Как видно из доказательства, это ограничение возникает, в первую очередь, из-за применения теоремы 1.3 из [5], в которой дается оценка наилучших равномерных рациональных приближений функций $f \in C_{A}$, удовлетворяющих условию $f^{\prime} \in E_{1}$. Что касается неравенства (3), то оно выполняется для существенно более широкого класса областей (подробности см. в [1], [8]).

В заключение отметим, что аналоги теоремы 2 для пространств Смирнова $E_{p}, 0<$ $p \leqslant 1$, получены нами в [8] (см. теоремы 3.3 и 3.4). Для пространств $E_{p}$ при $1<p<\infty$ нужный результат вытекает из обобщенной теоремы М. Рисса.

\section{СПИСОК ЦИТИРОВАННОЙ ЛИТЕРАТУРЫ}

[1] В. И. Данченко, Analysis Math., 16 (1990), 241-255. [2] А. А. Гончар, Л. Д. Григорян, Матем. сб., 99:4 (1976.), 634-638. [3] Л. Д. Григорян, Матем. сб., 100:1 (1976), 156164. [4] А. А. Пекарский, Матем. заметки, 31:3 (1982), 389-402. [5] А. А. Пекарский, Алгебра и анализ, 13:2 (2001), 165-190. [6] G. G. Lorentz, M. V. Golitschek, Y. Makovoz, Constructive Approximation. Advanced Problems, Springer, Berlin, 1996. [7] И. И. Привалов, Граничные свойства аналитических функиий, ГИТТЛ, М-Л., 1950. [8] А. А. Пекарский, Алгебра и анализ, 16:3 (2004), 143-170.

\section{А. А. Пекарский}

Белорусский государственный технологический университет

E-mail: pekarski@bstu.unibel.by 